\title{
Statistical Study on Cortical Sulci of Human Brains
}

Xiaodong Tao ${ }^{1,3}$, Xiao Han ${ }^{1}$, Maryam E. Rettmann ${ }^{2}$, Jerry L. Prince ${ }^{1,2,3}$, and Christos Davatzikos ${ }^{3}$

1 Electrical and Computer Engineering Johns Hopkins University, Baltimore, MD 21218, USA

xtao, xhan,prince@iron.ece.jhu.edu

2 Biomedical Engineering, Johns Hopkins University, Baltimore, MD 21218, USA rettmann@bme.jhu.edu

3 Department of Radiology, Johns Hopkins University, School of Medicine, Baltimore, MD 21287, USA

hristos@rad.jhu.edu

\begin{abstract}
A method for building a statistical shape model of sulci of the human brain cortex is described. The model includes sulcal fundi that are defined on a spherical map of the cortex. The sulcal fundi are first extracted in a semi-automatic way using an extension of the fast marching method. They are then transformed to curves on the unit sphere via a conformal mapping method that maps each cortical point to a point on the unit sphere. The curves that represent sulcal fundi are parameterized with piecewise constant-speed parameterizations. Intermediate points on these curves correspond to sulcal landmarks, which are used to build a point distribution model on the unit sphere. Statistical information of local properties of the sulci, such as curvature and depth, are embedded in the model. Experimental results are presented to show how the models are built.
\end{abstract}

\section{Introduction}

The cortex of the human brain is a thin convoluted surface comprised of gyri and sulci, which are folds oriented outwards and inwards, respectively. It is believed that many cortical sulci are linked to the underlying cytoarchitectonic and functional organization of the brain, although this relationship varies throughout the cortex and is not well understood at present. Recently, there has been great interest within the brain imaging community in developing image analysis methods for characterizing sulcal shapes. Such methods would have several applications. First, sulci are natural pathways to deeper brain structures in certain neurosurgical procedures. Therefore, the better understanding of their structures is important in neurosurgical planning [1]. Second, it has been suggested [2] that sulcal shapes are related to the underlying connectivity of the brain, since they are influenced by forces exerted by connecting fibers. Therefore, shape analysis of the sulci is important in understanding normal variability, as well as in studying developmental disorders or effects of aging. 
The third application of sulcal shape analysis is the primary focus of the work described in this paper. Specifically, sulci and gyri can serve as features used in spatial normalization algorithms. Spatial normalization is frequently used to map data to a stereotaxic coordinate system, by removing inter-subject morphological differences, thereby allowing for group analysis to be carried out. The 3D Talairach coordinate system has been extensively used in the brain mapping literature, but surface-based coordinate systems have also been proposed for studying the cortex, which has a surface topology [34].

In this paper we describe steps toward building a statistical shape model of major cortical sulci, by using the unit sphere as stereotaxic space. Sulci are projected onto the unit sphere via a conformal mapping procedure [56]. Our model captures inter-subject variability of the shape and depth of the sulci, and is intended for automatic labeling and spatial normalization of cortical surfaces extracted from magnetic resonance images.

Previous attempts to build statistical models of the sulci have relied on graphs constructed from 3D point-sets [17], on ribbons used to model the space between opposite sides of a sulcus 891011, or on curves located on the outer cortical surface [12. Related is the work in [413,14, in which sulci are not explicitly modeled, but they are spatially normalized via a curvature matching procedure that stretches individual surfaces into conformation with an average curvature map. Finally, related is also the work in 15], where manually drawn sulcal curves, located on the outer cortical surface, were spatially normalized via a robust matching algorithm.

In contrast to most of the previous work 1416, our sulcal model is comprised of sulcal fundi, the deepest parts of the sulci, which are treated as parameterized curves located on the unit sphere. Fundi are first found via a modified fast marching algorithm [17] applied on cortical surfaces extracted via the method reported in [18]. A conformal mapping algorithm [5,6] is then used to place the sulci on the unit sphere, which serves as the stereotaxic coordinate space. Statistics on the shape variability and on the depth of the fundi are then incorporated into this model. Our current model consists of seven sulci of the lateral surface of the right hemisphere.

\section{Methods}

In this section, we first describe the steps involved in constructing our statistical model of seven major sulcal fundi. We then describe preliminary work towards registering this model to label an individual brain's sulcal fundi. In the training stage, parameterized curves running along the fundi are built, using an extension of the fast marching algorithm. These curves are then transformed to the unit sphere via conformal mapping and are aligned via a Procrustes fit [19], resulting in a number of parameterized curves serving as training examples. From these curves we build a model that has two elements. First, an attribute vector 20] is attached to each point on a sulcal fundus. If it is rich enough, this attribute vector can distinguish different sulci, and hence facilitate the subsequent deformation 
and labeling process. Second, statistical shape variation of the fundi is captured via the principle eigenvectors of the covariance matrix [21].

\subsection{Spherical Representation of Brain Cortex}

The cortical surfaces used in this work are reconstructed from MR brain images using a largely automatic method reported in [18. Fuzzy segmentation, an isosurface algorithm and a deformable model are used to reconstruct the central layer of brain cortex with correct topology. The method has been validated both qualitatively and quantitatively in [18.

The brain cortex is a thin gray matter sheet and is topologically equivalent to a sphere when closed at the brain stem. This fact has motivated the work of several groups to mapping the cortical surface to a sphere, so that visualization of deep sulci is easier. The sphere can also play the role of a stereotaxic space, within which the location and size of sulcal fundi are normalized, allowing for the calculation of statistical parameters which can be used for automatic recognition. In this paper, we use the conformal mapping method in 6] to map cortical surfaces to the unit sphere in a standard way. The method was developed from the one initially proposed by Angenent et al 5 .

The conformal mapping method starts with a reconstructed cortical surface represented by a triangular mesh. A point is chosen on the top of the corpus callosum, which corresponds to the north pole after the cortical surface is mapped to the sphere. The whole cortical surface is then mapped to the complex plane using the technique described in [5]. The points on the complex plane are mapped to the unit sphere using an inverse stereographic projection to generate a spherical map of the original cortical surface. As it is pointed out in [6], the conformal spherical map of a cortical surface is not unique. This fact gives us the flexibility to select the map that minimizes the area distortion in the regions that contain the fundi of our model by adopting a similar technique used in [6] to minimize the overall area distortion. Instead of finding parameters that minimize the total area distortion, we find the parameters that minimize the area distortion in the regions of interest.

\section{$2.2 \quad$ Feature Extraction}

A sulcus is the region between two juxtaposed sides of a cortical fold. It is often modeled as a thin convolved ribbon embedded in 3D 89,1011. Sulcal fundi are $3 \mathrm{D}$ curves that lie on the deepest parts of the sulci and are regions of high curvature of the cortex. Because of the convoluted nature of the cortex, it is difficult to visualize sulcal fundi. Manually extracting them is an even more difficult task. For this reason, investigators have reported algorithms for obtaining line representations of sulci and gyri [22 23 24]. We adopt a similar strategy herein, by modeling fundi as parametric curves lying on the unit sphere. In order to build a training set of sulcal fundi, we use a semi-automatic approach based on the fast marching method on triangulated meshes [17]. Interaction is required 
by the algorithm in defining the initial, final, and intermediate points along the sulcal fundi.

The Fast Marching method is a numerical approach for solving the Eikonal equation [17:

$$
|\nabla T(\boldsymbol{x})| f(\boldsymbol{x})=1, \quad \boldsymbol{x} \in \mathcal{C}
$$

where $\mathcal{C}$ is a surface represented by rectangular orthogonal grids or triangulated meshes, $f(\boldsymbol{x})$ is a given non-negative function defined on $\mathcal{C}$ and $T(\boldsymbol{x})$ is the function to be solved. Consider the case where a monotonically advancing front is propagating with a speed $f(\boldsymbol{x})>0$, then $T(\boldsymbol{x})$ is the time for the front to cross the point $\boldsymbol{x}$ from its initial position. If the front propagates with unit speed over $\mathcal{C}$, i.e. $f(\boldsymbol{x}) \equiv 1, T(\boldsymbol{x})$ is the geodesic distance from point $\boldsymbol{x}$ to the initial front location. The fast marching method can be used to find the geodesic path between two points $A$ and $B$ on a triangulated surface by first solving Eq. 11 with the boundary condition $T(A)=0$, and then back tracking in the negative gradient direction of $T$ from $B$ [17. Because the surface is treated as a continuum, the geodesic path so constructed has a sub-grid resolution without dividing the grids in any fashion.

In order to extract sulcal fundi, we use the fact that the fundi have high curvatures and large depths. By setting proper speed terms $f(\boldsymbol{x})$, we can make the path calculated by the fast marching method favor trajectories that run along the sulcal fundi. In this work, we set the speed term $f(\boldsymbol{x})$ in Eq. (1) as follows (numbers are given in pixels, where one pixel is $0.9375 \mathrm{~mm}$ ):

$$
f(\boldsymbol{x})= \begin{cases}0.1 & \text { if } d(\boldsymbol{x})<1.5 \\ d(\boldsymbol{x}) & \text { if } 1.5 \leq d(\boldsymbol{x})<3.0, \quad \text { for } \boldsymbol{x} \in \mathcal{C} \\ \kappa_{m}^{2}(\boldsymbol{x})+3.0 & \text { if } d(\boldsymbol{x}) \geq 3.0\end{cases}
$$

Here, $d(\boldsymbol{x})$ is the geodesic depth at $\boldsymbol{x}$, which is defined as the geodesic distance between $\boldsymbol{x}$ and the outer surface of the brain obtained via a shrink wrapping procedure 2526]; $\kappa_{m}(\boldsymbol{x})$ is the mean curvature at $\boldsymbol{x}$. In the deep part of a sulcus, where $d(\boldsymbol{x})>3.0$, those points with high curvature will have high speed. This results in a curve running through points with high curvature. In the shallow part of a sulcus, where $1.5<d(\boldsymbol{x}) \leq 3.0$, the speed term is determined solely by the depth. Therefore in this region, the sulcal curves extracted by this algorithm favor points with large depth. In gyral regions, where $d(\boldsymbol{x})<1.5$, the speed term is set to be a small positive number, so that the curve can climb over the interruptions of a sulcus. The speed term so defined makes the resulting path favor trajectories running along sulcal fundi. The extracted sulcal fundi are 3-D curves represented by ordered lists of points on the surface, which are readily mapped onto the unit sphere via the computed conformal map.

The following definitions and notations are used in the following sections. A shape $\mathcal{S}$ is a collection of piecewise constant-speed curves $C_{i}(s), i=1, \cdots, k$, which are parameterized in the unit interval, $s \in[0,1]$. After discretization, each curve consists of a number of points, $c_{i j}=C_{i}\left(s_{j}\right)$, which are used in a point 
distribution model. We construct these curves so that points of the same parametric coordinate, $s$, correspond to roughly anatomically homologous regions. Therefore, we call $c_{i j}$ landmark points. The coordinate vector $\mathcal{V}$ of a shape $\mathcal{S}$ is a vector consisting of the coordinates of the landmark points of $\mathcal{S}$. It is arranged as $\mathcal{V}^{T}=\left[x_{1}, y_{1}, z_{1}, \cdots, x_{M}, y_{M}, z_{M}\right]$, where $M$ is the total number of landmark points of the shape. Crucial points are those landmark points in between which the speed of the curve's parameterization is constant; they are typically the end points of a sulcus, or intermediate points corresponding to sulcal intersections.

\subsection{Statistical Shape Model}

In order to build a statistical shape model of the sulcal fundi, it is necessary to explicitly specify the point correspondence between shapes, which is often difficult. In [14, the Iterative Closest Point algorithm was employed to fulfill this task as well as to bring the shapes into alignment. In our work, we first identify a number of crucial points, typically corresponding to the connections between different curves, such as the connection between the superior frontal sulcus and the pre-central sulcus. Since we are dealing with primary sulci that are relatively stable across subjects, the end points and connections are easy to identify. The crucial points are manually picked with reference to the sulcal segmentation [26], in which sulcal regions are segmented using a watershed method based on the geodesic depth. Each point on the cortex with a geodesic depth greater than a certain threshold is considered to be located on a sulcus. The watershed method is then used to group those sulcal points into regions. With the help of these sulcal regions, the end points of the sulci can be consistently identified. Once crucial points are picked, the curve segments between them are parameterized by arc length. In this way, point correspondence is established naturally between any two shapes.

Let $\mathcal{V}_{1}, \mathcal{V}_{2}, \cdots, \mathcal{V}_{N}$ be $N$ coordinate vectors extracted from $N$ brains. By applying the Procrustes fit, the shapes are brought into alignment so that they have the same size, same location and similar pose. The standard Procrustes fit for shapes on a 2D plane is as follows [21]19]: first, translate each shape so that its centroid coincides with the origin of the 2D plane; second, scale each translated shape so that the coordinate vector of the shape has unit norm; and finally, rotate each shape to minimize its distance to the mean shape. We extend this approach by applying it on shapes defined on the unit sphere. As it is in the planar case, we need 4 parameters to specify the location, size and pose of a shape on the unit sphere. They are $\theta$ and $\phi$ for location, $c$ for size and $\alpha$ for pose. The Procrustes fit on sphere is as follows:

1. Each shape is rotated so that its centroid is on the $z$-axis. This step is equivalent to the translation step in the planar case. The rotation is done by a coordinate system transformation that transforms the centroid of the shape $\left(x_{0}, y_{0}, z_{0}\right)$ into the north pole $(0,0,1)$. For each point $(x, y, z)$ on the unit sphere, the transformation can be expressed in matrix form as: 


$$
\left(\begin{array}{l}
x^{\prime} \\
y^{\prime} \\
z^{\prime}
\end{array}\right)=\left(\begin{array}{ccc}
\cos \theta \cos \phi \sin \theta \cos \phi & -\sin \phi \\
-\sin \theta & \cos \theta & 0 \\
\cos \theta \sin \phi & \sin \theta \sin \phi & \cos \phi
\end{array}\right)\left(\begin{array}{l}
x \\
y \\
z
\end{array}\right)
$$

where $\theta, \phi$ are the spherical coordinates of shape centroid $\left(x_{0}, y_{0}, z_{0}\right)$. The mean and the variance of the location of the shape centroids are calculated. They are denoted by $\bar{\theta}, \bar{\phi}, \sigma_{\theta}^{2}$ and $\sigma_{\phi}^{2}$, respectively. By performing this rotation, the patch of the sphere containing the curves being modeled is sitting around the north pole.

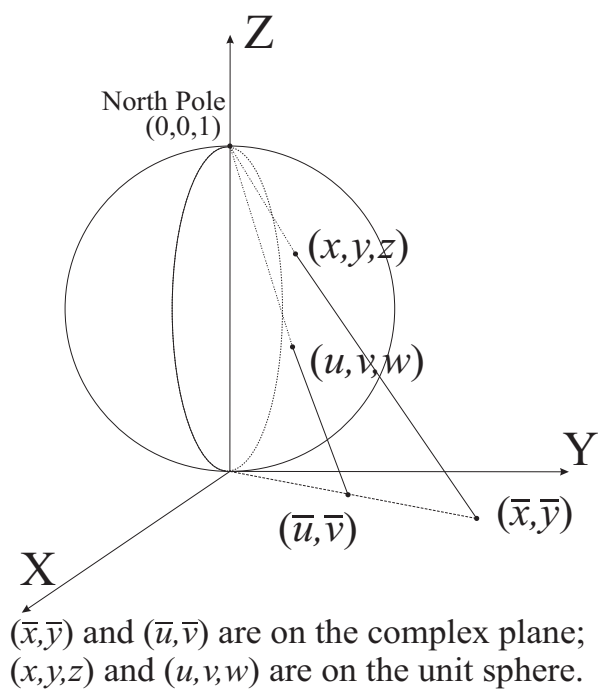

Fig. 1. Scaling a spherical patch, used for subsequent Procrustes fit.

2. The procedure generating the conformal map of the brain cortex enables us to scale the patch that contains the sulcal curves: 1) mapping the unit sphere to the complex plane via stereographic projection and therefore mapping the shape on the unit sphere to a shape on the complex plane; 2) scaling the shape on the complex plane as usual; and 3) mapping the scaled shape on the complex plane back to the unit sphere via inverse stereographic projection. The size of a shape is measured as the sum of the the great circle distances between each landmark point and the north pole. This procedure is illustrated in Fig. 1. A point on the unit sphere $(x, y, z)$ is first mapped to $(\bar{x}, \bar{y})$ on the complex plane using stereographic projection. $(\bar{x}, \bar{y})$ is then scaled by a factor of $c$ to be $(\bar{u}, \bar{v})=c(\bar{x}, \bar{y})$. Finally, $(\bar{u}, \bar{v})$ is mapped back to the sphere as $(u, v, w)$ via inverse stereographic projection. In this way, a spherical patch around the north pole is scaled. 
3. Shapes that are translated and scaled are then rotated around the $z$-axis so as to minimize the misalignment. This rotation is different from the rotation in Step 1. Here, the rotation changes the pose of the shape, while the rotation in Step 1 changes the location of the shape. Rotation around the $z$-axis by an angle $\alpha$ can be expressed in matrix form as:

$$
\left(\begin{array}{c}
u^{\prime} \\
v^{\prime} \\
w^{\prime}
\end{array}\right)=\left(\begin{array}{ccc}
\cos \alpha & -\sin \alpha & 0 \\
\sin \alpha & \cos \alpha & 0 \\
0 & 0 & 1
\end{array}\right)\left(\begin{array}{l}
u \\
v \\
w
\end{array}\right)
$$

After the shapes are brought into alignment, the statistics on the shapes are readily computed. The point distribution model consists of a mean shape and a number of eigenmodes of variation. With this model, any new shape can be approximated by its projection onto the model space.

In addition to the point distribution model, each landmark point is associated with an attribute vector, whose elements are the statistics of the local properties at that point. The attribute vectors can include a variety of shape attributes. Currently, we use depth and curvature at different scales. These attribute vectors capture the shape information in the neighborhood at different resolutions at each landmark point. For example, from the results shown in Fig. 6, we can see that the depth profiles along the central, pre-central, and post-central sulci are quite different. Therefore the attribute vectors can potentially help distinguish among different sulci, and hence facilitate automatic labeling.

\subsection{Registration}

The model built using the algorithm described above can be used to search for and label sulcal fundi in an unseen brain image. The registration stage is divided into two steps: linear and nonlinear. We have currently implemented a linear matching, which is used for initialization of the deformable model. In particular, the mean shape is put onto the spherical map of the unseen brain image. Then by searching for the best values for $\theta, \phi, c$ and $\alpha$ in the intervals obtained from the training stage, the best estimation for the sulcal fundi in the unseen image can be found and nonlinear registration can be performed thereafter using a hierarchical scheme 12 .

\section{Results and Discussion}

Experiments were conducted using 8 T1-weighted volumetric MR brain images. The images were pre-processed to correct the intensity inhomogeneity introduced by imaging devices, and to strip the irrelevant tissue such as skin, fat, and skull. They were then processed using the reconstruction method reported in [18] to extract the central layer of the cortical surfaces. In our work, a statistical model was built for the central, pre-central, post-central, superior frontal, inferior frontal, superior temporal, and circular insular sulci on the right hemisphere. 


\subsection{Extracting Sulcal Fundi}

Brain cortices were visualized using OpenDX. With the tools provided by the software, we picked the crucial points of each sulcus with reference to a brain atlas [27] and the sulcal segmentation results. For each individual sulcus on a brain cortex, we used the method described in Section 2.2 to compute the distance between each point $\boldsymbol{x}$ on the surface to the starting point of the sulcus, and then extracted the sulcal fundi by back-tracking in the negative gradient direction of the distance function starting from the end point of the sulcus. Because of the nature of our algorithm, the points on the sulcal fundi do not need to lie on vertices. Fig. 2 shows a central sulcus viewed from inside the brain. From the figure, it can be clearly seen that the extracted fundi are quite reliable. Fig. 4(a) shows the fundi of several sulci extracted from one brain image, and Fig. 4(b) shows the spherical map of 7 sulcal fundi of one brain on the unit sphere. After discretization of the resulting parametric curves, the total number of landmark points is 850 .

\subsection{Model}

Fig. 5(a) shows the sulci of 8 data sets after Procrustes fit. The mean shape and the most significant eigenvariation of the resulting model (see Sec. 2.3) are shown in Fig. 5(b). The thick curves are the mean position of the sulcal fundi and the thin ones show the eigenvariation at some landmark points with length equal to one standard deviation to each direction.

Figs. 6(a), (b), and (c) show the geodesic depth along the central, pre-central and post-central sulci of eight training brains. The comparison of the means and the standard deviations are shown in Fig. 6(d). As Fig. 6(d) shows, the depth profiles along different sulci are very different. We believe that as many such attributes are included in our model, different sulci will have sufficiently different attribute vectors to allow for robust deformation of the model.

From Fig. 6, it can be seen that there are some obvious properties of depth profiles for different sulci:

1. For the central sulcus, somewhere in the middle, there is a consistent decrease in depth; this part is likely to be the area of the pre-central knob.

2. Along the pre-central sulcus, there is a consistent interruption, partial or total, reflected by an abrupt decrease in depth. Fig. 6(d) shows that this interruption of the pre-central sulcus is very consistent across subjects, since the standard deviation of the depth is small in that region. This fact gives us confidence that the pre-central sulcus will be detected fairly easily, thereby making the detection of nearby sulci (central, post-central, superior frontal sulci) easier.

3. The deviation along the central sulcus is generally smaller than those of the pre-central and post-central sulci. 


\subsection{Linear Registration}

In order to label sulcal fundi in a new dataset, a linear registration was first performed, in order to initialize a deformation process. In this step, the mean shape obtained in Sec. 3.2 was put onto the new data set, then it was rotated and scaled so that it had the best size, position and pose in the sense that the local geometric properties at each landmark point fit the statistics of those obtained from the training set. Fig. 3 shows the result of the rigid registration on a new dataset. Currently, we are in the process of implementing a hierarchical deformation mechanism for this model, similar to the one described in [12 20]28].

\section{Conclusion}

In this paper, the sulcal fundi of a brain cortex are extracted from the brain cortical surface using a semi-automatic method. They are transformed to the unit sphere using a conformal mapping method and parameterized to be piecewise uniform-speed curves. A point distribution model is then built from them, in which each landmark point has some statistics on its location. Moreover, each landmark point has an associated attribute vector, which describes the local geometric properties of the brain. This model can be used to detect and label sulcal fundi on an unseen dataset. In our experiments, we used 8 brains as our training examples. Although this training set is very small, it gives us a clear idea of the consistency of the location and depth profile of several sulci. This statistical information is important in sulcal labeling. Future work includes non-rigid registration using the model built with a larger training set via the methodology presented in this paper. Other attributes will also be examined, which will help uniquely characterize sulci, and hence make the model more precise.

\section{Acknowledgments}

This work was partially supported by NIH grant R01AG14971, NIH contract N01AG32129, NIH grant R01NS37747 and NSF/ERC grant CISST\#9731748. The authors would like to acknowledge the Baltimore Longitudinal Study of Aging which provided the datasets.

\section{References}

1. J.-F. Mangin, V. Frouin, I. Bloch, J. Regis and J. Lopez-Krahe, "From 3D magnetic resonance images to structural representations of the cortex topography using topology preserving deformations," J. Math. Imag. Vis., vol. 5, pp. 297-318, Dec. 1995.

2. D. C. Van Essen and J. H. R. Maunsell, "Two dimensional maps of cerebral cortex," J. Comp. Neurol., vol. 191, no. 2, pp. 255-281, 1980. 


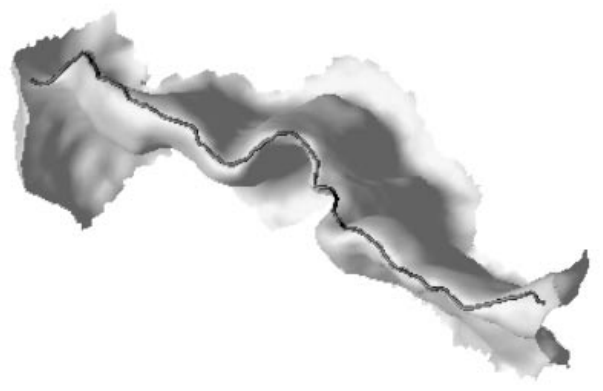

Fig. 2. A central sulcus viewed from inside the brain. Black curve is the sulcal fundus extracted using our method. It divides the entire sulcus into anterior and posterior banks.

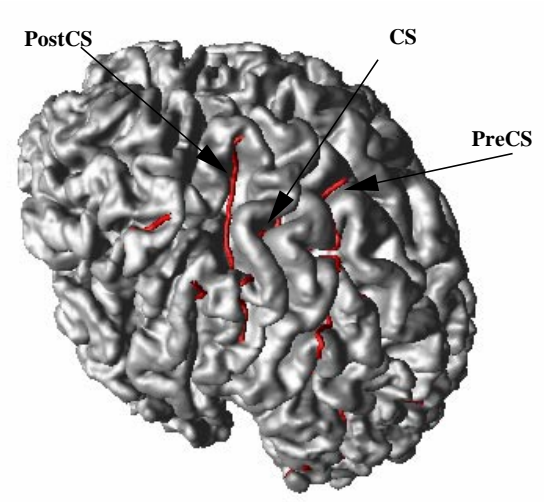

(a)

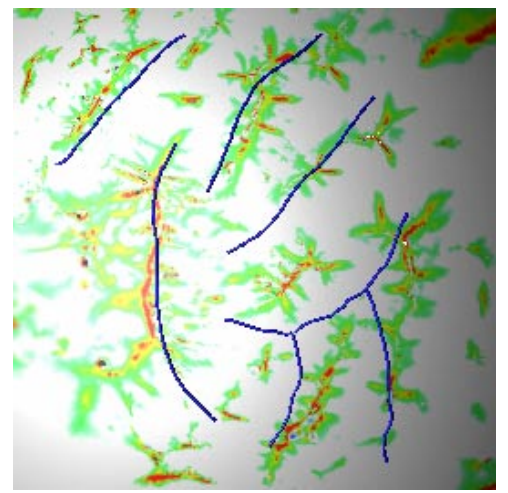

Fig. 3. The spherical patch containing the sulcal curves being modeled, after linear registration with a new image. The mean shape has been scaled, translated and rotated to have the best match with the input image.



(b)

Fig. 4. (a) Sulcal fundi on a brain cortical surface. (b) The same fundi after conformal mapping of the cortex onto the unit sphere. 


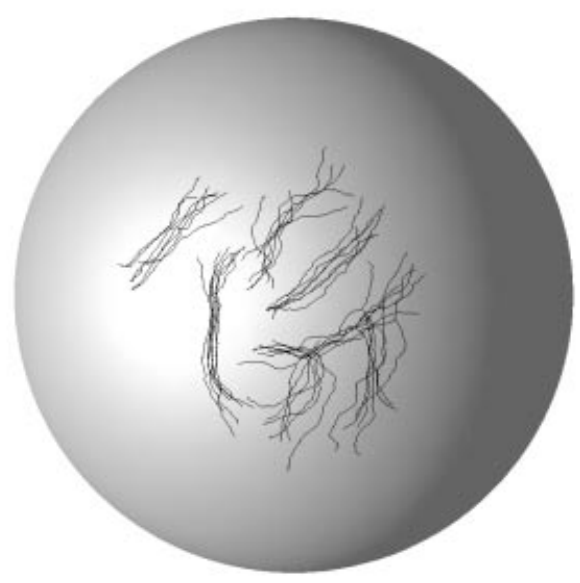

(a)

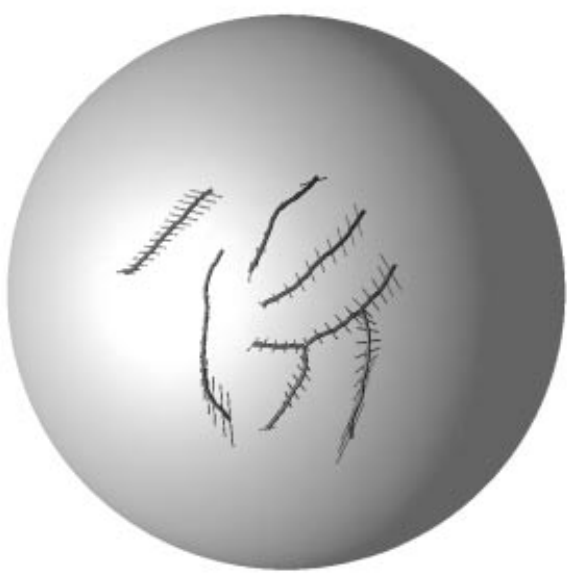

(b)

Fig. 5. (a) Sulcal fundi of 8 subjects after aligned using Procrustes fit. (b) The mean shape and the most significant eigenmode (1 standard deviation to each side from the mean).
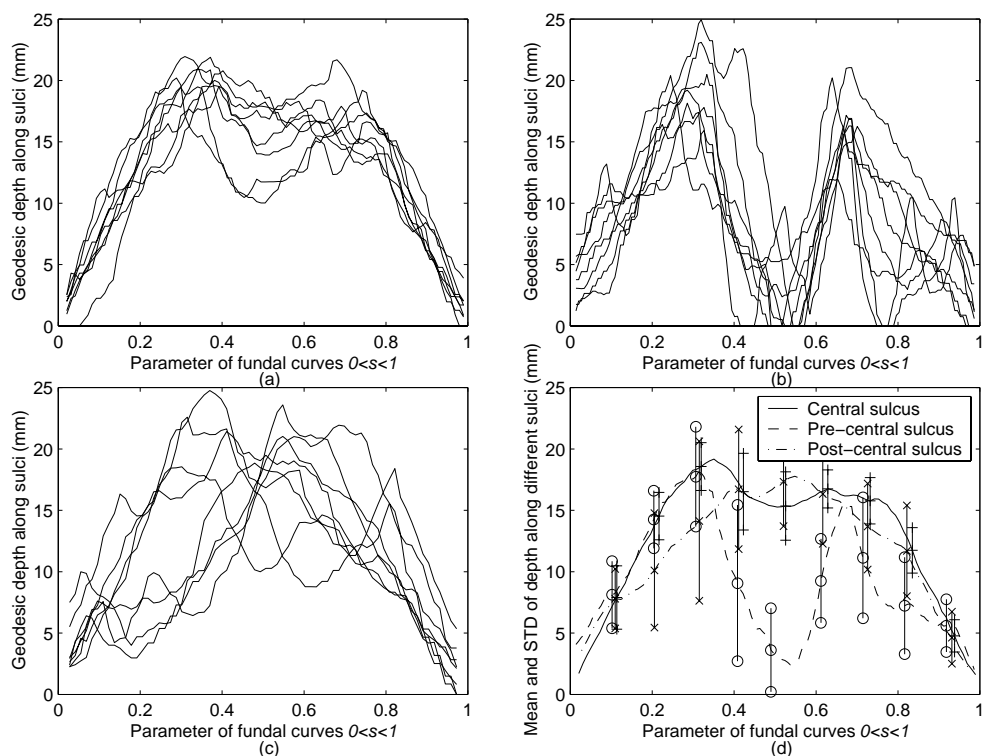

Fig. 6. Depth profile along the central (a), pre-central (b) and post-central (c) sulci of 8 subjects. (d) The mean depth and standard deviation at several points along each sulcus. 
3. H. A. Drury, D. C. Van Essen, C. H. Anderson, C. W. Lee, T. A. Coogan and J. W. Lewis, "Computerized mappings of the cerebral cortex: A multiresolution flattening method and a surface-based coordinate system", J. Cogn. Neurosci. , vol. 8, no. 1, pp. 1-28, Jan. 1996.

4. A. M. Dale, B. Fischl and M. I. Sereno, "Cortical surface-based analysis I: Segmentation and surface reconstruction," Neuroimage, vol. 9, no. 2, pp. 179-194, Feb. 1999.

5. S. Angenent, S. Haker, A. Tannenbaum and R. Kikinis, "On the Laplace-Beltrami Operator and Brain Surface Flattening", IEEE Trans. Med. Imag., vol. 18, no. 8, pp. 700-711, Aug. 1999.

6. D. Tosun and J. L. Prince, "A Hemispherical Map for the Human Brain Cortex", Proc. of SPIE Conf. on Medical Imaging, to appear, 2001.

7. G. Lohmann, F. Kruggel and D. Y. von Cramon, "Automatic detection of sulcal bottom lines in MR images of human brain", Proc. of Information Processing in Medical Imaging (IPMI), pp. 369-374, 1997.

8. M. Vaillant and A. Davatzikos, "Finding parametric representations of the cortical sulci using an active contour model", Medical Image Analysis, vol. 1, no. 4, pp. 295-315, 1997.

9. G. Le Goualher, D. L. Collins, C. Barillot and A. C. Evans, "Automatic Identification of Cortical Sulci Using a 3D Probabilistic Atlas", Proc. of Medical Image Computing and Computer-Assisted Intervention (MICCAI), pp. 509-517, Springer, 1998.

10. P. M. Thompson, C. Schwartz and A. W. Toga, "High-resolution random mesh algorithms for creating a probabilistic 3D surface atlas of the human brain", Neuroimage, vol. 3, no. 1, pp. 19-34, Feb. 1996.

11. X. Zeng, L. H. Staib, R. T. Schultz, H. Tagare, L. Win and J. S. Duncan, "A New Approach to 3D Sulcal Ribbon Finding from MR Images", Proc. of Medical Image Computing and Computer-Assisted Intervention (MICCAI), pp. 148-157, Springer, 1999.

12. M. Vaillant and A. Davatzikos, "Hierarchical Matching of Cortical Features for Deformable Brain Image Registration", Proc. of Information Processing in Medical Imaging (IPMI), pp. 182-195, Springer, 1999.

13. B. Fischl, M. I. Sereno, and A. M. Dale, "Cortical surface-based analysis II: Inflation, flattening, and a surface-based coordinate system," Neuroimage, vol. 9, pp. 195-207, 1999.

14. A. Caunce and C. J. Taylor, "Using local geometry to build 3D sulcal models", Proc. of Information Processing in Medical Imaging (IPMI), pp. 196-209, Springer, 1999.

15. H. Chui, J. Rambo, J. Duncan, R. Schultz and A. Rangarajan, "Registration of Cortical Anatomical Structures via Robust 3D Point Matching", Proc. of Information Processing in Medical Imaging (IPMI), pp. 168-181, Springer, 1999.

16. N. Royackkers, M. Desvignes, H. Fawal and M. Revenu, "Detection and Statistical Analysis of Human Cortical Sulci", Neuroimage, vol. 10, pp. 625-641, 1999.

17. R. Kimmel and J. A. Sethian, "Computing Geodesic Paths on Manifolds", Proceedings of National Academy of Sciences, vol. 95, no. 15, pp. 8431-8435, 1998.

18. C. Xu, D. L. Pham, M. E. Rettmann, D. N. Yu, and J. L. Prince, "Reconstruction of the human cerebral cortex from magnetic resonance images," IEEE Trans. Med. Imag., vol. 18, no. 6, pp. 467-480, June 1999.

19. I. L. Dryden and K. V. Mardia, Statistical Shape Analysis, JOHN WILEY \& SONS, New York, NY, 1998. 
20. D. Shen and C. Davatzikos, "Adaptive-Focus Statistical Shape Model for Segmentation of 3D MR Structures", Proc. of Medical Image Computing and ComputerAssisted Intervention (MICCAI), pp. 206-215, Springer, 2000.

21. T. F. Cootes and C. J. Taylor, "The Use of Active Shape Models for Locating Structures in Medical Images", Proc. of Information Processing in Medical Imaging (IPMI), pp. 33-47, Springer, 1993.

22. J. Declerck, G. Subsol, J.P. Thirion and N. Ayache, "Automatic retrieval of anatomical structure in 3D medical images", Proc. of CVRMed'95, pp. 153-162, 1995.

23. N. Khaneja, M. I. Miller and U. Grenander, "Dynamic programming generation of curves on brain surfaces", IEEE Trans. on Pattern Anal. Machine Intell., vol. 20, no. 11, pp. 1260-1265, Nov. 1998.

24. G. Lohmann, "Extracting Line Representations of Sulcal and Gyral Patterns in MR Images of the Human Brain", IEEE Trans. on Medical Imaging, vol. 17, no. 6, pp. 1040-1048, Dec. 1998.

25. M. E. Rettmann, C. Xu, D. L. Pham and J. L. Prince, "Automated Segmentation of Sulcal Regions", Proc. of Medical Image Computing and Computer-Assisted Intervention (MICCAI), pp. 158-165, Springer, 1999.

26. M. E. Rettmann, X. Han, C. Xu and J. L. Prince, "Watersheds on the cortical surface for automated sulcal segmentation", Proc. of the Workshop on Mathematical Methods in Biomedical Image Analysis, pp. 20-27, 2000.

27. H. M. Duvernoy, "The Human Brain: Surface, Three-Dimensional Sectional Anatomy with MRI, and Blood Supply", Springer, 1999.

28. D. Shen and C. Davatzikos, "An adaptive-focus deformable model using statistical and geometric information", IEEE Trans. on Pattern Anal. Machine Intell., vol. 22, no. 8, pp. 906-913, Aug. 2000. 